\title{
Treatment Choices Based on OncotypeDx in the Breast Oncology Care Setting
}

\author{
Teri L. Malo, ${ }^{1}$ Isaac Lipkus, ${ }^{2}$ Tobi Wilson, ${ }^{3}$ Hyo S. Han,, 5 \\ Geza Acs, ${ }^{4,5,6}$ and Susan T. Vadaparampil ${ }^{1,4}$ \\ ${ }^{1}$ Health Outcomes and Behavior Program, Division of Cancer Prevention and Control, Moffitt Cancer Center, \\ 12902 Magnolia Drive, MRC-CANCONT, Tampa, FL 33612, USA \\ ${ }^{2}$ School of Nursing, Duke University, Medical Center, P.O. Box 3322, Durham, NC 27710, USA \\ ${ }^{3}$ Department of Surgery, Feinberg School of Medicine, Northwestern University, 251 East Huron Street Galter 3-150, \\ Chicago, IL 60611, USA \\ ${ }^{4}$ Department of Oncologic Sciences, College of Medicine, University of South Florida, 12901 Bruce B. Downs Boulevard, MDC 44, \\ Tampa, FL 33612, USA \\ ${ }^{5}$ Department of Women's Oncology, Moffitt Cancer Center, 12902 Magnolia Drive, Tampa, FL 33612, USA \\ ${ }^{6}$ Department of Anatomic Pathology, Moffitt Cancer Center, 12902 Magnolia Drive, Tampa, FL 33612, USA
}

Correspondence should be addressed to Susan T. Vadaparampil, susan.vadaparampil@moffitt.org

Received 29 March 2012; Accepted 17 May 2012

Academic Editor: Suzanne C. O’Neill

Copyright ( $) 2012$ Teri L. Malo et al. This is an open access article distributed under the Creative Commons Attribution License, which permits unrestricted use, distribution, and reproduction in any medium, provided the original work is properly cited.

\begin{abstract}
Introduction. This study aimed to evaluate whether Oncotype Dx test results predict receipt of adjuvant chemotherapy in breast cancer patients who received an OncotypeDx recurrence score (RS). Materials and Methods. Pathology records were used to identify breast cancer patients who had OncotypeDx testing between December 2004 and January $2009(n=118)$. Patient sociodemographic information, tumor characteristics, RS, and treatment-specific data were collected via chart review. RS was classified as follows: low (RS $\leq 17)$, intermediate $(\mathrm{RS}=18-30)$, or high ( $\mathrm{RS} \geq 31$ ). Bivariate analyses were conducted to investigate the relationship between adjuvant chemotherapy receipt and each sociodemographic and clinical characteristic; significant sociodemographic and clinical variables were included in a multivariable logistic regression model. Results. In multivariable analysis controlling for tumor size, histologic grade, and nuclear grade, only RS remained significantly associated with chemotherapy uptake. Relative to low RS, an intermediate (adjusted odds ratio [AOR], 21.24; 95\% confidence interval [CI], 3.62-237.52) or high (AOR, 15.07; 95\% CI, 1.28-288.21) RS was associated with a greater odds of chemotherapy uptake. Discussion. Results indicate that RS was significantly associated with adjuvant chemotherapy uptake, suggesting that OncotypeDx results were used to inform treatment decision making, although it is unclear if and how the information was conveyed to patients.
\end{abstract}

\section{Introduction}

An estimated 226,870 new cases of breast cancer are expected to be diagnosed in 2012 and account for about 29\% of all new cancer cases among US women [1]. Adjuvant chemotherapy is one treatment option for breast cancer patients that is used because of its potential to reduce the risk of breast cancer recurrence and mortality [2]; however, not all patients benefit from adjuvant chemotherapy [3]. Moreover, chemotherapy may be detrimental to quality of life given its potential to produce toxicities, including myelosuppression $[2]$.
The quality of breast cancer care can be improved by informing treatment selection based on individual patient genomic risk profiles [4]; however, to realize the greatest benefits, advances in predictive models that inform treatment decisions must be accepted and used by healthcare providers and patients [5]. One example of predictive modeling that is rapidly moving into the breast oncology care setting is OncotypeDx testing $[4,6]$. Based on the expression of 21 genes obtained from tumor tissue, OncotypeDx testing calculates the risk of breast cancer distant recurrence (i.e., the chance of breast cancer returning as metastatic disease) in patients with estrogen receptor (ER) positive early breast 
cancer treated with adjuvant endocrine therapy and predicts the clinical benefit with additional adjuvant chemotherapy. Given that approximately $75 \%$ of breast cancers are ER positive and $61 \%$ of those cases are lymph node (LN) negative [7], many women with breast cancer may be qualified and benefit from OncotypeDx testing.

Although there are clinical guidelines to identify patients who would derive the greatest benefit from OncotypeDx testing [6], relatively few studies have been conducted to examine the impact of test results on treatment decisions. Some research has indicated that OncotypeDx recurrence score (RS) results have impacted receipt of chemotherapy, including a study of 276 patients who were newly diagnosed with breast cancer between 2005 and 2009 [8]. After controlling for Nottingham Prognostic Index, adjuvant online mortality risk, progesterone receptor (PR) status, and medical oncologists' blinded recommendation for adjuvant chemotherapy, only RS and patient age at diagnosis were significantly associated with receipt of adjuvant chemotherapy. Other studies have linked RS results to changes in adjuvant chemotherapy plans [8-12]. For instance, one study conducted from 2004 to 2006 examined the impact of RS on 29 patients with ER positive and LN negative breast cancer [9]. Results showed that RS changed chemotherapy plans for 9 patients such that 7 of 13 patients for whom chemotherapy was recommended did not receive it, and 2 of 16 patients received chemotherapy after initial recommendations against it.

Though some research has pointed to an association between RS and receipt of adjuvant chemotherapy, currently only one published study collected data beyond 2008 [8]. The current study serves to replicate these findings in another population, with the primary purpose of evaluating whether OncotypeDx test results predict receipt of adjuvant chemotherapy in a cohort of consecutive patients with breast cancer who received an OncotypeDx RS.

\section{Materials and Methods}

The study population was comprised of patients treated at Moffitt Cancer Center, a large National Cancer Institutedesignated comprehensive cancer center in the southeastern US. Approximately $60 \%$ of all patients seen at Moffitt come from the surrounding seven county catchment area, with the remainder of patients coming from other Florida counties, states, and countries. Upon Institutional Review Board approval, Moffitt Cancer Center surgical pathology records were used to identify patients meeting the following criteria (1) diagnosed with breast cancer, and (2) had OncotypeDx testing between December 2004 (the year in which the test was approved by the U.S. Food and Drug Administration) and January 2009 (the year in which chart reviews were completed). Based on this information, medical records were reviewed retrospectively to collect patient sociodemographic information, tumor characteristics, Oncotype Dx RS, and treatment-specific data. Chart abstractions were performed by a study team member after training from the principal investigator. The senior study coordinator reviewed $10 \%$ of data files to assess the accuracy of collected data. Finally, a medical oncologist reviewed the summarized data and identified a subset ( $\sim 10 \%$ of charts) for additional review to ensure accuracy.

2.1. Measures. Sociodemographic data included age; marital, parental, and menopause status; race/ethnicity; and family history of breast cancer (present/absent). Clinical characteristics included breast cancer stage; tumor size; LN status; histology; modified combined histologic (Nottingham) grade; nuclear grade; human epidermal growth factor receptor-2 (HER2), ER, PR, and angiolymphatic invasion status; and RS. Regarding RS, patients were classified into one of three groups based on cut points: low (RS $\leq 17)$, intermediate (RS $=18-30)$, or high $(\mathrm{RS} \geq 31)$ [13]. The primary outcome variable, receipt of adjuvant chemotherapy, was based on grouping women into those who had chemotherapy versus those who did not. This comparison was selected as it most closely reflects the primary treatment decision influenced by OncotypeDx results $[6,13]$.

2.2. Data Analyses. Bivariate analyses were used to investigate the relationship between the primary outcome variable, receipt of adjuvant chemotherapy, and the sample sociodemographic and clinical characteristics. Pearson Chi-square or Fisher's Exact tests were used to study relationships with each categorical variable of interest; a $t$-test was conducted to examine differences in age and RS by chemotherapy uptake group. Analyses used two-tailed tests of significance with the significance level set at $P<0.05$.

In an effort to maintain an acceptable case-to-variable ratio, independent variables included in the final multivariable logistic regression model were selected based on variables significant in the bivariate analyses. Odds ratios (OR) and their 95\% confidence interval (CI) were estimated from the logistic regression model. Given the variables' small expected cell counts, an exact analysis was conducted. Analyses were performed using the SAS 9.1 statistical software package (SAS Institute Inc, Cary, North Carolina).

\section{Results}

Chemotherapy status was unknown for 8 patients; data for the remaining 118 patients were used for analyses. Patient demographic and clinical characteristics are presented in Table 1. Patients' mean age was 56.3 years $(\mathrm{SD}=11.0$; range: 33-84). Most participants were married or living with a partner $(70.3 \%)$, White $(84.8 \%)$, had children $(72.0 \%)$, and were perimenopausal/postmenopausal $(63.6 \%)$. Just over half $(52.5 \%)$ had no family history of breast cancer.

Most patients had stage I breast cancer (80.5\%), a tumor size $>1.0 \mathrm{~cm}(78.8 \%)$, and were LN negative $(95.8 \%)$. The majority $(84.8 \%)$ had invasive ductal carcinoma (IDC), and over half $(56.8 \%)$ of the tumors showed intermediate histologic grade. The largest proportion of patients had a nuclear grade of $2(47.5 \%)$. Most patients' tumors were HER2 negative (94.9\%), ER positive (99.2\%), PR positive (89.0\%), 
TABLE 1

(a) Patient demographic and clinical characteristics by chemotherapy group $(N=118)^{\mathrm{a}}$

\begin{tabular}{|c|c|c|c|c|}
\hline \multirow[b]{2}{*}{ Characteristics } & \multirow[b]{2}{*}{$\begin{array}{c}\text { Total } \\
(N=118) n(\%)\end{array}$} & \multicolumn{2}{|c|}{ Chemotherapy } & \multirow[b]{2}{*}{$P^{\mathrm{b}}$} \\
\hline & & $\begin{array}{c}\text { Yes } \\
(n=35) n(\%)\end{array}$ & $\begin{array}{c}\text { No } \\
(n=83) n(\%)\end{array}$ & \\
\hline \multicolumn{5}{|l|}{ Demographic } \\
\hline Age in years mean (SD) & $56.3(11.0)$ & $54.1(10.4)$ & $57.3(11.1)$ & .1513 \\
\hline Marital status & & & & .0968 \\
\hline Married/living with partner & $83(70.3)$ & $28(80.0)$ & $55(66.3)$ & \\
\hline Other & $33(28.0)$ & $6(17.1)$ & $27(32.5)$ & \\
\hline Parental status & & & & .6933 \\
\hline Children & $85(72.0)$ & $24(68.6)$ & $61(73.5)$ & \\
\hline No children & $28(23.7)$ & $9(25.7)$ & $19(22.9)$ & \\
\hline Menopause status & & & & .2037 \\
\hline Premenopause & $38(32.2)$ & $14(40.0)$ & $24(28.9)$ & \\
\hline Perimenopause/postmenopause & $75(63.6)$ & $19(54.3)$ & $56(67.5)$ & \\
\hline Race/ethnicity & & & & .6003 \\
\hline White & $100(84.8)$ & $29(82.9)$ & $71(85.5)$ & \\
\hline Other & $17(14.4)$ & $6(17.1)$ & $11(13.3)$ & \\
\hline Family history & & & & .5314 \\
\hline Absent & $62(52.5)$ & $17(48.6)$ & $45(54.2)$ & \\
\hline Present & $55(46.6)$ & $18(51.4)$ & $37(44.6)$ & \\
\hline \multicolumn{5}{|l|}{ Clinical } \\
\hline Breast cancer stage & & & & .0595 \\
\hline I & $95(80.5)$ & $25(71.4)$ & $70(84.3)$ & \\
\hline II A & $19(16.1)$ & $8(22.9)$ & $11(13.3)$ & \\
\hline II B & $2(1.7)$ & $0(0.0)$ & $2(2.4)$ & \\
\hline III/III B & $2(1.7)$ & $2(5.7)$ & $0(0.0)$ & \\
\hline Tumor size (mean in $\mathrm{cm}$ ) & 1.6 & 1.8 & 1.5 & .0694 \\
\hline$\leq .5 \mathrm{~cm}$ & $3(2.5)$ & $1(2.9)$ & $2(2.4)$ & $.0462^{*}$ \\
\hline $0.6-1.0 \mathrm{~cm}$ & $22(18.6)$ & $2(5.7)$ & $20(24.1)$ & \\
\hline$>1.0 \mathrm{~cm}$ & $93(78.8)$ & $32(91.4)$ & $61(73.5)$ & \\
\hline Lymph node & & & & 1.0000 \\
\hline Positive & $4(3.4)$ & $1(2.9)$ & $3(3.6)$ & \\
\hline Negative & $113(95.8)$ & $34(97.1)$ & $79(95.2)$ & \\
\hline Histology & & & & .1542 \\
\hline IDC & $100(84.8)$ & $33(94.3)$ & $67(80.7)$ & \\
\hline ILC & $7(5.9)$ & $2(5.7)$ & $5(6.0)$ & \\
\hline Mixed & $9(7.6)$ & $0(0.0)$ & $9(10.8)$ & \\
\hline Other & $2(1.7)$ & $0(0.0)$ & $2(2.4)$ & \\
\hline Histologic grade & & & & $.0160^{*}$ \\
\hline Low & $33(28.0)$ & $7(20.0)$ & $26(31.3)$ & \\
\hline Intermediate & $67(56.8)$ & $18(51.4)$ & $49(59.0)$ & \\
\hline High & $17(14.4)$ & $10(28.6)$ & $7(8.4)$ & \\
\hline
\end{tabular}

(b) Patient demographic and clinical characteristics by chemotherapy group

\begin{tabular}{lcccc}
\hline Characteristics & Total & Chemotherapy & No \\
& $(N=118)$ & $\begin{array}{c}\text { Yes } \\
(n=35)\end{array}$ & $P^{\mathrm{a}}$ \\
\hline Nuclear grade & & & & $.0004^{*}$ \\
1 & $6(5.1)$ & $1(2.9)$ & $5(6.0)$ & \\
2 & $56(47.5)$ & $8(22.9)$ & $48(57.8)$ & \\
3 & $24(20.3)$ & $14(40.0)$ & $10(12.1)$ & \\
\hline
\end{tabular}


(b) Continued.

\begin{tabular}{|c|c|c|c|c|}
\hline \multirow[b]{2}{*}{ Characteristics } & \multicolumn{4}{|c|}{ Chemotherapy } \\
\hline & $\begin{array}{c}\text { Total } \\
(N=118)\end{array}$ & $\begin{array}{c}\text { Yes } \\
(n=35)\end{array}$ & $\begin{array}{c}\text { No } \\
(n=83)\end{array}$ & $P^{\mathrm{a}}$ \\
\hline HER2 & & & & .3598 \\
\hline Positive & $6(5.1)$ & $3(8.6)$ & $3(3.6)$ & \\
\hline Negative & $112(94.9)$ & $32(91.4)$ & $80(96.4)$ & \\
\hline ER & & & & .2966 \\
\hline Positive & $117(99.2)$ & $34(97.1)$ & $83(100.0)$ & \\
\hline Negative & $1(0.9)$ & $1(2.9)$ & $0(0.0)$ & \\
\hline $\mathrm{PR}$ & & & & .0560 \\
\hline Positive & $105(89.0)$ & $28(80.0)$ & $77(92.8)$ & \\
\hline Negative & $13(11.0)$ & $7(20.0)$ & $6(7.2)$ & \\
\hline Angiolymphatic invasion & & & & .1092 \\
\hline Absent & $86(72.9)$ & $23(65.7)$ & $63(75.9)$ & \\
\hline Present & $20(17.0)$ & $9(25.7)$ & $11(13.3)$ & \\
\hline Recurrence score (mean) & 19.0 & 26.4 & 15.9 & $<.0001^{*}$ \\
\hline Low & $68(57.6)$ & $4(11.4)$ & $64(77.1)$ & $<.0001^{*}$ \\
\hline Intermediate & $37(31.4)$ & $24(68.6)$ & $13(15.7)$ & \\
\hline High & $13(11.0)$ & $7(20.0)$ & $6(7.2)$ & \\
\hline
\end{tabular}

${ }^{a}$ Percentages may not total 100 as a result of rounding or missing data, or both.

${ }^{\mathrm{b}}$ A chi-square or Fisher's exact test was used for categorical variables, and an independent samples $t$-test was used to compare means for the age, tumor size, and recurrence risk score variables.

and did not show angiolymphatic invasion (72.9\%). The mean RS was 19.0 and the largest proportion of patients had a low RS (57.6\%).

In bivariate analyses, tumor size, histologic grade, nuclear grade, and RS were significantly associated with uptake of adjuvant chemotherapy (Table 1). Compared to patients who did not receive chemotherapy, those who received chemotherapy had a greater proportion of tumor size $>1.0 \mathrm{~cm}$ (91.4\% versus $73.5 \%)$, high histologic grade $(28.6 \%$ versus $8.4 \%)$, nuclear grade of $3(40.0 \%$ versus $12.1 \%)$, intermediate $(68.6 \%$ versus $15.7 \%)$ and high $(20.0 \%$ versus $7.2 \%)$ RS category, and higher mean RS (26.4 versus 15.9$)$. In multivariable analysis controlling for tumor size, histologic grade, and nuclear grade, only RS remained statistically significantly associated with chemotherapy receipt (Table 2 ). Relative to those with a low RS, those with an intermediate (adjusted odds ratio [AOR], 21.24; 95\% CI, 3.62-237.52) or high (AOR, 15.07; 95\% CI, 1.28-288.21) RS had a greater odds of chemotherapy uptake.

\section{Discussion}

Study results indicate that RS was significantly associated with adjuvant chemotherapy uptake, suggesting that in our sample of female breast cancer patients who underwent OncotypeDx testing, the results of this gene assay were likely being used to inform treatment decision making, although it is unclear if and how the information was conveyed to patients. RS score was a significant predictor of chemotherapy uptake after controlling for more standard clinicopathological markers used to guide treatment
TABLE 2: Logistic regression for uptake of adjuvant chemotherapy $(n=85)$.

\begin{tabular}{lc}
\hline Variable & Adjusted odds ratio $(95 \%$ confidence interval) \\
\hline Tumor size & Reference \\
$\leq .5 \mathrm{~cm}$ & $0.21(0.00-32.74)$ \\
$0.6-1.0 \mathrm{~cm}$ & $1.24(0.03-138.62)$ \\
$>1.0 \mathrm{~cm}$ & \\
Histologic grade & Reference \\
Low & $0.62(0.05-8.16)$ \\
Intermediate & $0.33(0.01-9.71)$ \\
High & \\
Nuclear grade & Reference \\
1 & $0.74(0.01-122.90)$ \\
2 & $2.42(0.02-563.30)$ \\
3 & \\
Recurrence score* & Reference \\
Low & $21.24(3.62-237.52)$ \\
Intermediate & $15.07(1.28-288.21)$ \\
High &
\end{tabular}

selection, specifically tumor size, LN status, histologic grade, and nuclear grade. These results are consistent with earlier research that has shown that RS was associated with whether patients received adjuvant chemotherapy or not [8-12].

Adjuvant chemotherapy is recommended for individuals with RS scores in the high-risk category, whereas it is unlikely to benefit individuals in the low-risk category $[13,14]$. 
In our study, patients with a low RS were least likely to receive adjuvant chemotherapy ( $94 \%$ of low-risk patients did not receive chemotherapy). This finding is consistent with research showing that RS affects treatment decision-making for low-risk patients [15] and is similar to the Ademuyiwa study [8], which reported that $91 \%$ of patients with a low RS did not receive chemotherapy.

Although National Comprehensive Cancer Network guidelines recommend chemotherapy for individuals with high RS, in our patient population, only $54 \%$ of individuals with high RS received chemotherapy. This percentage is lower than that of the Ademuyiwa study [8], which reported that $96 \%$ of patients with high RS received chemotherapy. For our sample of patients in these higher risk categories, the receipt of chemotherapy is likely multifactorial (based on coexisting clinicopathological features). Due to the small sample sizes in this study, we were unable to more fully investigate the patterns of treatment choice based on these multiple factors.

Of interest was the finding that one ER negative patient and four LN positive patients received an RS, even though the test has been validated for ER positive and LN negative breast cancer patients at the time OncotypeDx testing was performed. The ER negative patient was HER2 negative and T1cN0. All LN positive patients were HER2 negative with primary tumor categories ranging from one to four. Additionally, there were six HER2 positive patients who received an RS, one of whom was T1aN0, four were T1bN0, and one was T1cN0. It is possible that the RS was felt to be of utility for these patients, and perceived as an additional measure of breast cancer recurrence risk to support a decision about adjuvant chemotherapy, as was noted in one patient chart.

Most of the published literature regarding the use of RS to guide treatment selection focuses on physicians' use of this information in guiding treatment selection $[8,10]$, with limited evaluation of the patient's role in the decision to pursue a therapy. Factors that patients may consider in their treatment decisions include side effects that can adversely affect quality of life, patient preference for participation in treatment decision-making, and understanding of the results; the latter may be particularly critical where greatest uncertainty exists, that is, for patients with an intermediate RS. A preference for an active role in decision-making among those presented with OncotypeDx results is related to health literacy, such that women with high-health literacy prefer a more active role in decision-making, whereas lowhealth literacy is related to preference for more shared and passive decision-making [16]. Our group recently completed a retrospective cross-sectional survey of a subset of the patients $(n=64)$ included in the present study and found that women incorrectly answered approximately half of a series of 14 items evaluating knowledge about RS [17]. Another similarly designed study of 77 breast cancer patients found that one-third of participants did not fully understand discussions related to RS [18]. Although not a focus of this study, it would be of interest to investigate how these variables interact to guide treatment planning for patients in the higher-risk RS categories.
To date, much of the research on OncotypeDx results' association with treatment decision-making has occurred in an academic setting. Some research conducted with an inner-city population suggests that Oncotype Dx results may influence chemotherapy treatment decisions in this setting [19]. In this sample of 47 women who underwent OncotypeDx testing, 5\% of women with a low RS and $100 \%$ of women with a high RS received chemotherapy. The results for women with a low RS are aligned with the current study and Ademuyiwa study [8], and results for women with a high RS are similar to the Ademuyiwa study. More research needs to be conducted with larger samples of women from populations outside of the academic setting.

This study has several limitations. First, the confidence intervals for the RS categories were wide, thus estimates may be imprecise. This is likely an effect of the relatively small sample size. Second, although several clinicopathological and demographic variables were included in the study, the small sample sizes limited our ability to further elucidate trends among individuals in the higher-risk RS categories who did not receive adjuvant chemotherapy. Third, family history was recorded in the patient's chart based on patient self-report and, like other self-report data, may be inaccurate; however, some research on the concordance between self-report of family breast cancer history and cancer confirmation sources (e.g., state tumor registry) suggests a sensitivity ranging from $61 \%$ to $95 \%[20,21]$. Fourth, as the study was retrospective, we were unable to assess definitively whether treatment planning was changed as a result of the availability of the RS score and whether patients themselves were able to use the RS to aid their treatment decision. Also, we did not account for the possible role that comorbidity may have played in the uptake of chemotherapy. Given that higher comorbidity has been linked to a decreased likelihood of receiving chemotherapy among women aged 55 and older [22], it is possible that comorbid conditions may also have been associated with chemotherapy among older women in the current study. Another limitation is the relatively heterogenous population with regard to race/ethnicity. Although reflective of the patient population at this institution, findings may not be generalizable to other races and ethnicities. Finally, some patients may have received chemotherapy after the data collection period ended and this change in chemotherapy receipt status was not reflected in the analyses.

In summary, the current study offers relatively recent data to support results of previous research documenting a change in treatment selection based on the use of the RS [8-12]. The literature remains in need of studies investigating the variables associated with a patient's use of this valuable health information, including the potential role of comorbidities, as well as studies conducted with populations outside of the academic setting. This paper has important implications for prevention, such that women with a lower risk of breast cancer recurrence as determined by OncotypeDx testing may be able to avoid the potential toxicity associated with chemotherapy. 


\section{Conflict of Interests}

Regarding potential conflict of interests, Dr. Acs serves on the Speakers' Bureau for Genomic Health, Inc. and Clarient, Inc. The other authors report no conflicts.

\section{Acknowledgment}

This paper was supported by the Miles for Moffitt Foundation Funds.

\section{References}

[1] R. Siegel, D. Naishadham, and A. Jemal, "Cancer statistics, 2012,” CA Cancer Journal for Clinicians, vol. 62, no. 1, pp. 1029, 2012.

[2] M. Yang, S. Rajan, and A. M. Issa, "Cost effectiveness ofgene expression profiling for early stage breast cancer: a decisionanalytic model," Cancer. In press.

[3] N. Ishibe, S. Schully, A. Freedman, and S. D. Ramsey, "Use of Oncotype DX in women with node-positive breast cancer," PLoS Currents, vol. 3, article RRN1249, 2011.

[4] R. E. Ellsworth, D. J. Decewicz, C. D. Shriver, and D. L. Ellsworth, "Breast cancer in the personal genomics era," Current Genomics, vol. 11, no. 3, pp. 146-161, 2010.

[5] L. Wideroff, K. A. Phillips, G. Randhawa et al., "A health services research agenda for cellular, molecular and genomic technologies in cancer care," Public Health Genomics, vol. 12, no. 4, pp. 233-244, 2009.

[6] L. Harris, H. Fritsche, R. Mennel et al., "American society of clinical oncology 2007 update of recommendations for the use of tumor markers in breast cancer," Journal of Clinical Oncology, vol. 25, no. 33, pp. 5287-5312, 2007.

[7] W. F. Anderson, N. Chatterjee, W. B. Ershler, and O. W. Brawley, "Estrogen receptor breast cancer phenotypes in the Surveillance, Epidemiology, and End Results database," Breast Cancer Research and Treatment, vol. 76, no. 1, pp. 27-36, 2002.

[8] F. O. Ademuyiwa, A. Miller, T. O'Connor et al., “The effects of oncotype DX recurrence scores on chemotherapy utilization in a multi-institutional breast cancer cohort," Breast Cancer Research and Treatment, vol. 126, no. 3, pp. 797-802, 2011.

[9] L. R. Henry, A. Stojadinovic, S. M. Swain, S. Prindiville, R. Cordes, and P. W. Soballe, "The influence of a gene expression profile on breast cancer decisions," Journal of Surgical Oncology, vol. 99, no. 6, pp. 319-323, 2009.

[10] J. Asad, A. F. Jacobson, A. Estabrook et al., "Does oncotype DX recurrence score affect the management of patients with earlystage breast cancer?" American Journal of Surgery, vol. 196, no. 4, pp. 527-529, 2008.

[11] R. Oratz, D. Paul, A. L. Cohn, and S. M. Sedlacek, "Impact of a commercial reference laboratory test recurrence score on decision making in early-stage breast cancer," Journal of Oncology Practice, vol. 3, no. 4, pp. 182-186, 2007.

[12] S. S. Lo, P. B. Mumby, J. Norton et al., "Prospective multicenter study of the impact of the 21-gene recurrence score assay on medical oncologist and patient adjuvant breast cancer treatment selection," Journal of Clinical Oncology, vol. 28, no. 10, pp. 1671-1676, 2010.

[13] S. Paik, S. Shak, G. Tang et al., "A multigene assay to predict recurrence of tamoxifen-treated, node-negative breast cancer," New England Journal of Medicine, vol. 351, no. 27, pp. 28172826, 2004.
[14] S. Paik, S. Shak, and G. Tang, "Expression of the 21 genes in the Recurrence Score assay and prediction of clinical benefits in the tamoxifen NSABP study B-14 and chemotherapy in the NSABP study B-20," Breast Cancer Research and Treatment, vol. 88, supplement 1, pp. S15-S24, 2004.

[15] H. Liang, A. Burfsky, B. Lembersky, P. Rastogi, and V. Vogel, "A retrospective analysis of the impact of OncotypeDX low recurrence score results on treatment decisions in a single academic breast cancer center," Breast Cancer Research and Treatment, vol. 106, supplement S105, abstract, 2007.

[16] S. C. O’Neill, N. T. Brewer, S. E. Lillie et al., "Women's interest in gene expression analysis for breast cancer recurrence risk," Journal of Clinical Oncology, vol. 25, no. 29, pp. 4628-4634, 2007.

[17] I. M. Lipkus, S. T. Vadaparampil, P. B. Jacobsen, and C. A. Miree, "Knowledge about genomic recurrence risk testing among breast cancer survivors," Journal of Cancer Education, vol. 26, no. 4, pp. 664-669, 2011.

[18] J. P. Tzeng, D. Mayer, A. R. Richman et al., "Women's experiences with genomic testing for breast cancer recurrence risk," Cancer, vol. 116, no. 8, pp. 1992-2000, 2010.

[19] A. A. Guth, S. Fineberg, K. Fei, R. Franco, and N. Bickell, "Utilization of Oncotype DX to predict chemotherapy use in an inner-city population," Journal of Clinical Oncology, vol. 29, abstract e11131, 2011.

[20] P. L. Mai, A. O. Garceau, B. I. Graubard et al., "Confirmation of family cancer history reported in a population-based survey," Journal of the National Cancer Institute, vol. 103, no. 10, pp. 788-797, 2011.

[21] N. Qureshi, B. Wilson, P. Santaguida et al., "NIH state-ofthe-science conference: family history and improving health," Evidence Report/Technology Assessment 186, AHRQ Publication no. 09-E016, Agency for Healthcare Research and Quality, Rockville, Md, USA, 2009, Prepared by the McMaster University Evidence-based Practice Center, under Contract No. 290-2007-10060-I.

[22] A. Hawfield, J. Lovato, D. Covington, and G. Kimmick, "Retrospective study of the effect of comorbidity on use of adjuvant chemotherapy in older women with breast cancer in a tertiary care setting," Critical Reviews in Oncology/Hematology, vol. 59, no. 3, pp. 250-255, 2006. 


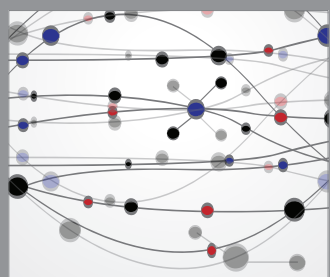

The Scientific World Journal
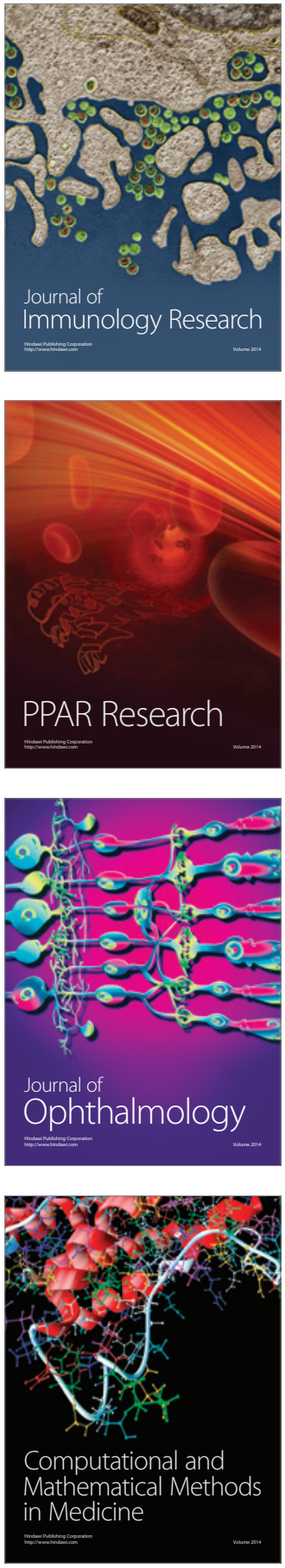

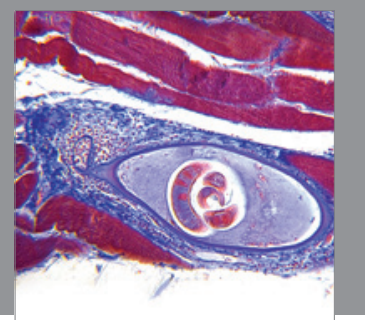

Gastroenterology

Research and Practice
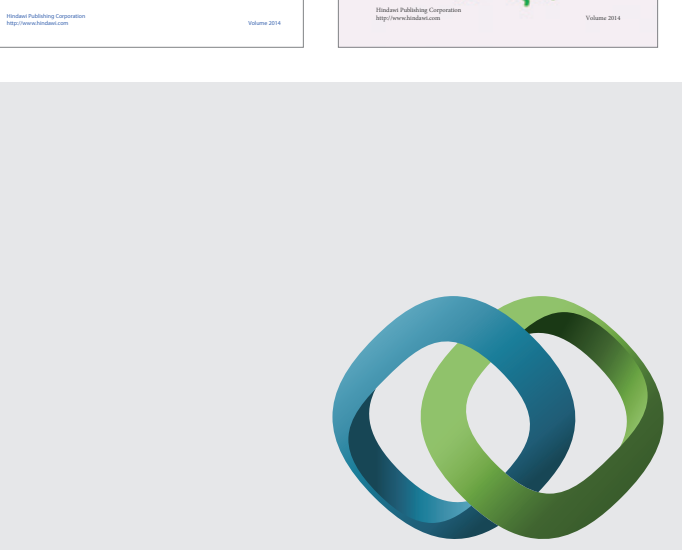

\section{Hindawi}

Submit your manuscripts at

http://www.hindawi.com
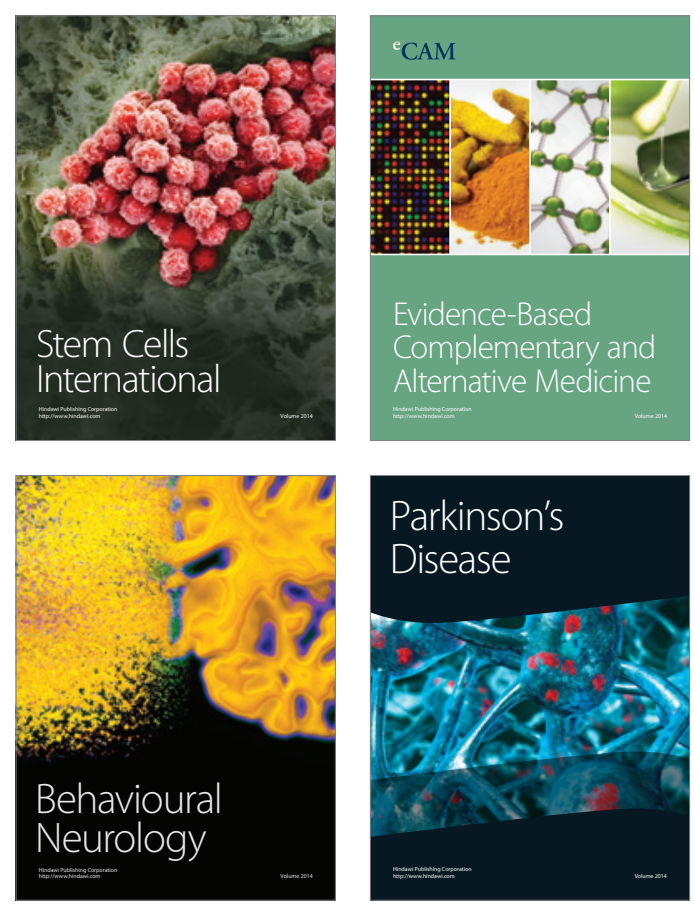

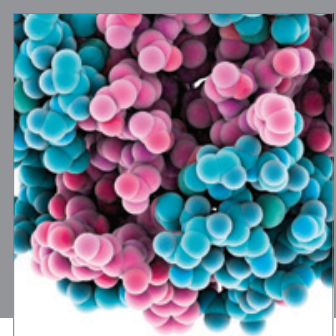

Journal of
Diabetes Research

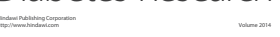

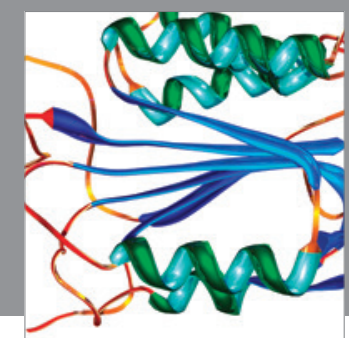

Disease Markers
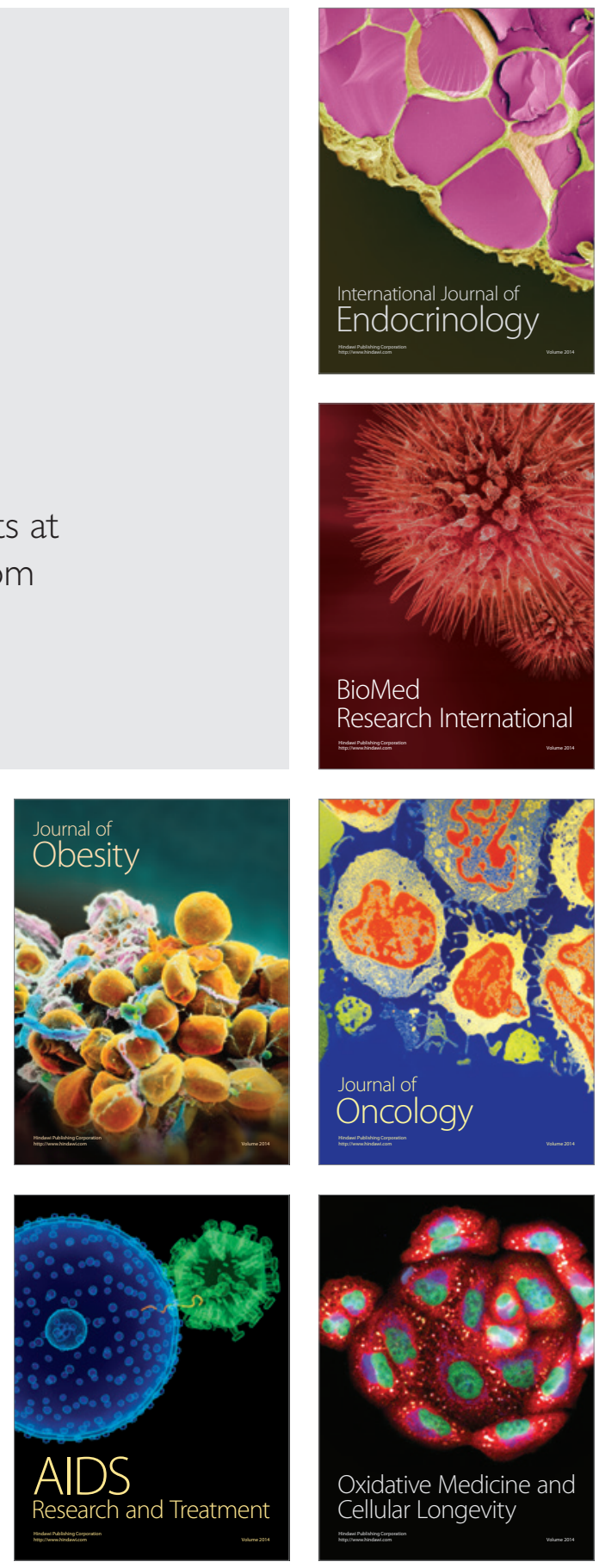